\title{
Equivalence among different formalisms in the Tsallis entropy framework
}

\author{
A.M. Scarfone \\ Istituto Nazionale di Fisica della Materia (CNR-INFM) and Dipartimento di \\ Fisica, Politecnico di Torino, Corso Duca degli Abruzzi 24, 10129 Torino, Italy. \\ T. Wada \\ Department of Electrical and Electronic Engineering, \\ Ibaraki University, Hitachi, Ibaraki, 316-8511, Japan.
}

\begin{abstract}
In a recent paper [Phys. Lett. A 335, 351 (2005)] the authors discussed the equivalence among the various probability distribution functions of a system in equilibrium in the Tsallis entropy framework. In the present letter we extend these results to a system which is out of equilibrium and evolves to a stationary state according to a nonlinear Fokker-Planck equation. By means of time-scale conversion, it is shown that there exists a "correspondence" among the self-similar solutions of the nonlinear Fokker-Planck equations associated with the different Tsallis formalisms. The time-scale conversion is related to the corresponding Lyapunov functions of the respective nonlinear Fokker-Planck equations.
\end{abstract}

Key words: Tsallis entropy, nonlinear Fokker-Planck equation, escort probability, Lyapunov function.

PACS: 05.20.Dd, 05.70.Ln, 05.90.+m, 65.40.Gr

\section{Introduction}

In the last decades some generalized entropies, different from the BoltzmannGibbs one, have been proposed in literature to study the statistical mechanics properties of complex systems with asymptotic free-scale behavior. Among the various proposals, the Tsallis' entropy [1]

$$
S_{q}[p]=\frac{1}{1-q} \int\left(p(v)^{q}-p(v)\right) d v,
$$


is a paradigm.

After its introduction in 1988, it has been fruitfully employed in the investigation of statistical systems characterized by long-rang interactions and time-persistent memory effects. Among the many, we quote astrophysical and cosmological systems, nuclear systems, low-dimensional chaotic systems, selforganized critical systems, turbulence. It is also widely employed in geological, biological and social-economical sciences (see [2] for a currently update bibliography).

As observed in [3], the original proposal [1] introduced the Lagrange multiplier associated with the linear mean energy in an unsatisfied manner so that some important properties necessary for a well stated theory were lacked. This insight induced in [3,4] to reformulate the original formalism (hereinafter we call it the modified 1st-formalism) based on the definition of the linear mean value given by

$$
A^{(1)}=\int A(v) p^{(1)}(v) d v .
$$

In [5,6] alternative definitions of the mean value were introduced, and the authors proposed the 2 nd-formalism based on the un-normalized $q$-mean value

$$
A^{(2)}=\int A(v)\left(p^{(2)}(v)\right)^{q} d v
$$

which unfortunately has the drawback that $\langle 1\rangle^{(2)} \neq 1$ and the 3rd-formalism based on the escort probability mean value

$$
A^{(3)}=\frac{\int A(v)\left(p^{(3)}(v)\right)^{q} d v}{\int\left(p^{(3)}(v)\right)^{q} d v} .
$$

More recently, in [7,8] the optimal Lagrange multiplier formalism (OLMformalism) were introduced, where for the same physical input of the 3rdformalism, the constraint in the MaxEnt procedure is written as the centeredform

$$
\int A(v)\left(p^{(\mathrm{OLM})}(v)\right)^{q} d v-A^{(\mathrm{OLM})} \int\left(p^{(\mathrm{OLM})}(v)\right)^{q} d v=0
$$

The 1st- and 3rd-formalisms have the disadvantage of dealing with self-referential expressions for the distribution functions which originate computational difficulties in some cases. Differently, the 2nd- and the OLM-formalisms produce distribution functions that are not self-referential though the mean values are still self-referential (see [9] for a comparison between these two formalisms). Throughout all these alternative formulations, in [10,11], another formalism 
based on the entropy $S_{2-q}[p]$ and the linear averages (hereinafter $(2-q)$ formalism) has been developed. In particular, in [12] it has been shown that the $(2-q)$-formalism produces a non self-referential expression both in the distribution and in the mean values. (For a comparison of some physically relevant quantities in the different formalisms see Appendix A).

In [13], the entropy $S_{2-q}[p]$ and its equilibrium distribution have been employed to show the equivalence among the different formulations of the Tsallis theory. The same $(2-q)$-formalism plays an important role in the discussion of the present work. For this reason, and also to fix the notations used in this paper, we shortly collect hereinafter the main results obtained in [13].

According to the following relations

$$
\begin{aligned}
& \beta^{(1)}=(2-q) \beta_{2-q}^{(1)}\left(\bar{Z}_{2-q}^{(1)}\right)^{q-1} \\
& \gamma^{(1)}=\frac{1}{1-q}-\frac{2-q}{1-q}\left(\bar{Z}_{2-q}^{(1)}\right)^{q-1}-(2-q) \beta_{2-q}^{(1)}\left(\bar{Z}_{2-q}^{(1)}\right)^{q-1} U_{q}^{(1)}
\end{aligned}
$$

the equilibrium distribution obtained in the $(2-q)$-formalism

$$
p(v)=\alpha \exp _{q}\left(-\gamma^{(1)}-\beta^{(1)} \epsilon(v)\right)
$$

with $\alpha=(2-q)^{1 /(q-1)}$ and $\exp _{q}(x)=[1+(1-q) x]^{1 /(1-q)}$ is the $q$-deformed exponential, can be rewritten in the form

$$
p^{(1)}(v)=\frac{1}{\bar{Z}_{2-q}^{(1)}} \exp _{q}\left(-\beta_{2-q}^{(1)}\left(\epsilon(v)-U_{q}^{(1)}\right)\right),
$$

with $\left(\bar{Z}_{2-q}^{(1)}\right)^{q-1}=\int\left(p^{(1)}(v)\right)^{2-q} d v$, where, by replacing $2-q$ with $q$, we obtain the probability distribution function of the modified 1st-formalism [4]. In Eq. (1.8), $\gamma^{(1)}$ and $\beta^{(1)}$ are the Lagrange multipliers related, respectively, to the normalization $1=\int p(v) d v$ and to the linear mean energy $U_{q}=\int \epsilon(v) p(v) d v$ in the $(2-q)$-formalism, as well as $\beta_{2-q}^{(1)}$ is related to the Lagrange multiplier associated to the linear mean energy $U_{q}^{(1)}=\int \epsilon(v) p^{(1)}(v) d v$ in the 1st-formalism. Equivalently, the same expression (1.8) can be transformed in

$$
p^{(3)}(v)=\frac{1}{\bar{Z}_{q}^{(3)}} \exp _{q}\left(-\beta_{q}^{(3)}\left(\epsilon(v)-U_{q}^{(3)}\right)\right)
$$

corresponding to the distribution function derived in the 3rd-formalism [6], where $\beta^{(3)}=\beta_{q}^{(3)}\left(\bar{Z}_{q}^{(3)}\right)^{1-q}$ is the Lagrange multiplier of the escort mean energy $U_{q}^{(3)}=\frac{\int \epsilon(v)\left(p^{(3)}(v)\right)^{q} d v}{\int\left(p^{(3)}(v)\right)^{q} d v}$ and $\left(\bar{Z}_{q}^{(3)}\right)^{1-q}=\int\left(p^{(3)}(v)\right)^{q} d v$. 
The expressions (1.9) and (1.10) are equivalents, in the sense that $p^{(1)}(v) \equiv$ $p^{(3)}(v)$, if the conditions

$$
\beta^{(1)}=(2-q) \beta^{(3)}\left(\bar{Z}_{q}^{(3)}\right)^{2(q-1)}
$$

and

$$
U_{q}^{(3)}=U_{q}^{(1)}-\frac{2-q}{(1-q) \beta^{(1)}}\left[\left(\bar{Z}_{q}^{(3)}\right)^{q-1}-\left(\bar{Z}_{2-q}^{(1)}\right)^{q-1}\right]
$$

are fulfilled. (See also [14 for an alternative discussion on the equivalence among these different formalisms).

In a previous tentative to establish a correspondence among the various formulations described above, it has been introduced the escort entropy [6] 15]

$$
S_{q}^{(\mathrm{E})}[p]=\frac{1}{1-q}\left\{\frac{\int p^{(\mathrm{E})}(v, t) d v}{\left[\int\left(p^{(\mathrm{E})}(v, t)\right)^{1 / q} d v\right]^{q}}-1\right\} .
$$

This entropy is derived from $S_{q}[p]$ by using the transformation

$$
p^{(3)}(v)=\frac{\left(p^{(\mathrm{E})}(v)\right)^{1 / q}}{\int\left(p^{(\mathrm{E})}(v)\right)^{1 / q} d v},
$$

where distributions $p^{(3)}(v)$ and $p^{(\mathrm{E})}(v)$ came, respectively, from the entropy $S_{q}[p]$ constrained by the escort mean value $U_{q}^{(3)}$ and from the entropy $S_{\tilde{q}}^{(\mathrm{E})}(p)$, with $\tilde{q}=1 / q$, constrained by linear mean value $U_{\tilde{q}}^{(\mathrm{E})}=\int \epsilon(v) p^{(\mathrm{E})}(v) d v$.

In the following, we are concerned with self-similar solutions of a nonlinear Fokker-Planck equation (NFPE) derived from the $S_{2-q}[p]$ entropy with a linear drift. This kinetic equation, and some related versions, are widely considered in the literature [16,17,18] to study anomalous diffusion processes like fractal media diffusion or diffusion in a non-Newtonian fluid. Self-similar solutions corresponding to these NFPEs were also investigated in 1920,21 because their relevance in the description of several phenomenologies in condensed matter physics.

The purpose of the present work is twofold.

Firstly, we extend the discussion held in [13] to the case of a system out of equilibrium. This is performed in the next section 2, where we show that the self-similar solutions derived in the $(2-q)$-formalism are equivalent to those of the same kinetic equations obtained by employing the modified 1st-formalism and 3rd-formalism. 
Secondly, in section 3, we show that, by means of a time-scale conversion, there is a "correspondence" among the (self-similar) solutions of the NFPE derived in the $(2-q)$-formalism and the (self-similar) solutions of the NFPE derived in the escort-formalisms. As a by product, it is shown that there exists a "correspondence" with the (self-similar) solutions of the NFPE derived in the 3rd-formalism with a time dependent diffusion coefficient. To establish a relationship among different NFPEs is useful not only in the framework of the statistical mechanics based on the Tsallis entropy but in general with the purpose to classify the various kinetic equations in equivalence class such that any equation belonging to the same class describes substantially the same physical process. The conclusions are given in section 4 whilst a comparison among the different formalisms discussed in this paper is reported in the Appendix A.

\section{Nonlinear Fokker-Planck equation in the $(2-q)$-formalism}

In order to describe our problem we need to relate the production of entropy to a Fokker-Planck equation describing the kinetics of the system. This issue has been discussed in literature by several authors, both in a very general nonlinear kinetic framework [22,23,24] and, more specifically, in the Tsallis entropy framework [21,25,26].

Quite generally, the NFPE associated with a given entropy $S[p]$ can be obtained from the relation

$$
\frac{\partial}{\partial t} p(v, t)=-\frac{\partial}{\partial v} J(v, t)
$$

and assuming for the expression of the nonlinear current

$$
J(v, t)=-p(v, t) \frac{\partial}{\partial v}\left(\frac{\delta \mathcal{L}}{\delta p}\right) .
$$

The quantity $\partial(\delta \mathcal{L} / \delta p) / \partial v$ is the thermodynamic force, where $\mathcal{L}[p]$ is the Lyapunov functional for the given problem [27].

By posing

$$
\mathcal{L}[p]=\mathcal{L}_{q}^{(1)}[p] \equiv U_{q}^{(1)}-D^{(1)} S_{2-q}[p],
$$

where

$$
S_{2-q}[p]=\int \frac{(p(v, t))^{2-q}-p(v, t)}{q-1} d v
$$


is the Tsallis entropy in the $(2-q)$-formalism and $D^{(1)}$ is the constant diffusion coefficient, we obtain the following NFPE

$$
\frac{\partial}{\partial t} p(v, t)=\frac{\partial}{\partial v}\left[-h(v) p(v, t)+D^{(1)} \frac{\partial}{\partial v}(p(v, t))^{2-q}\right]
$$

where

$$
h(v)=-\frac{\partial \epsilon(v)}{\partial v}
$$

is the drift coefficient. Equation (2.5) differs, in $q \rightarrow 2-q$, from the kinetics equation derived in [20,21,22] from entropy $S_{q}[p]$. The same Eq. (2.5) has been first proposed in [25] and after reconsidered by different authors [28,29,30,31]. In the following, we assume $\epsilon(v)=\frac{1}{2} m v^{2}$ with $m>0$, which deals with a specific but very common expression for the drift coefficient, corresponding to the Uhlenbeck-Ornstein process in the $q \rightarrow 1$ limit. Without loss of generality, we can pose $m=1$. With this choice for $\epsilon(v)$, Eq. (2.5) admits self-similar solutions [1925, 32]. In the $(2-q)$-formalism such solutions are obtained by introducing the ansatz

$$
p(v, t)=\alpha \exp _{q}\left(-\gamma^{(1)}(t)-\beta^{(1)}(t) \frac{1}{2} v^{2}\right) .
$$

In this way, we derive the following system of differential equations for the unknown quantities $\gamma^{(1)}(t)$ and $\beta^{(1)}(t)$ :

$$
\begin{aligned}
& \frac{d}{d t} \gamma^{(1)}(t)+\left[1-(1-q) \gamma^{(1)}(t)\right]\left(1-D^{(1)} \beta^{(1)}(t)\right)=0 \\
& \frac{d}{d t} \beta^{(1)}(t)-(3-q) \beta^{(1)}(t)\left(1-D^{(1)} \beta^{(1)}(t)\right)=0
\end{aligned}
$$

Moreover, accounting for the constraints on the normalization and the linear mean energy, which give the relations 1

$$
\gamma^{(1)}(t)=\ln _{2-q}\left(\alpha_{1} \sqrt{U_{q}^{(1)}(t)}\right), \quad \beta^{(1)}(t)=\alpha_{2}\left(U_{q}^{(1)}(t)\right)^{\frac{q-3}{2}}
$$

1 Hereinafter, for the sake of simplicity, we assume $1<q<\frac{5}{3}$ to guarantee the convergence of all integrals on the full real axe $(-\infty,+\infty)$. The results can be straightforward generalized to $0<q<1$, accounting for the cut-off condition on the integration interval. 
with

$$
\alpha_{1}=\alpha \sqrt{2 \pi \frac{5-3 q}{q-1}} \frac{\Gamma\left(\frac{1}{q-1}-\frac{1}{2}\right)}{\Gamma\left(\frac{1}{q-1}\right)}, \quad \alpha_{2}=\frac{\alpha_{1}^{q-1}}{5-3 q},
$$

from Eqs. (2.8) or (2.9) we obtain the further differential equation

$$
\frac{\partial}{\partial t} \ln U_{q}^{(1)}(t)+2\left[1-\alpha_{2} D^{(1)}\left(U_{q}^{(1)}(t)\right)^{\frac{q-3}{2}}\right]=0,
$$

describing the time evolution of the mean energy.

Solutions of Eqs. (2.8), (2.9) and (2.12) are given by

$$
\begin{aligned}
& \gamma^{(1)}(t)=\gamma_{0}-\left[1-(1-q) \gamma_{0}\right] \ln _{q}\left(\frac{\beta^{(1)}(t)}{\beta_{0}}\right)^{\frac{1}{3-q}}, \\
& \beta^{(1)}(t)=\beta_{0}\left[\left(1-\beta_{0} D^{(1)}\right) e^{(q-3) t}+\beta_{0} D^{(1)}\right]^{-1}, \\
& U_{q}^{(1)}(t)=U_{0}\left[\left(1-\mu_{0} D^{(1)}\right) e^{(q-3) t}+\mu_{0} D^{(1)}\right]^{\frac{2}{3-q}},
\end{aligned}
$$

with $\mu_{0} \equiv \beta_{0}=\alpha_{2} U_{0}^{\frac{q-3}{2}}, \gamma_{0}=\ln _{2-q}\left(\alpha_{1} \sqrt{U_{0}}\right)$ and $U_{0}=U_{q}^{(1)}(0)$. At equilibrium, $t \rightarrow t_{\mathrm{e}}=+\infty$, from Eq. (2.14) we obtain the relation $\beta_{\mathrm{e}}^{(1)} D^{(1)}=1$, where $\beta_{\mathrm{e}}^{(1)} \equiv \beta^{(1)}\left(t_{\mathrm{e}}\right)$, which means, as already known [23,26], that the quantity $1 / D^{(1)}$ can be identified with the Lagrange multiplier related to the mean energy $U_{q}^{(1)}$.

Differently, by using in Eq. (2.5) the ansatz

$$
p^{(1)}(v, t)=\frac{1}{\bar{Z}_{2-q}^{(1)}(t)} \exp _{q}\left(-\beta_{2-q}^{(1)}(t)\left(\frac{1}{2} v^{2}-U_{q}^{(1)}(t)\right)\right)
$$

for the modified 1st-formalism, we obtain the following set of equations

$$
\frac{d}{d t} \ln X_{q}^{(1)}(t)+2 f_{q}^{(1)}(t)=0
$$

where $X_{q}^{(1)}(t)$ means $\left(\bar{Z}_{2-q}^{(1)}(t)\right)^{2}, 1 / \beta_{2-q}^{(1)}(t)$ and $U_{q}^{(1)}(t)$, respectively, with

$$
f_{q}^{(1)}(t)=1-D^{(1)} \beta^{(1)}(t)
$$


and we posed $\beta^{(1)}(t)=(2-q) \beta_{2-q}^{(1)}(t)\left(\bar{Z}_{2-q}^{(1)}(t)\right)^{q-1}$, according to Eq. (1.6). By means of the relations (1.6) and (1.7) the system (2.17) is equivalent to the system (2.8), (2.9) and (2.12). Correspondingly, its solutions

$$
\left(\bar{Z}_{2-q}^{(1)}(t)\right)^{2} \beta_{2-q}^{(1)}(t)=\frac{\left(z_{2-q}^{(1)}\right)^{2}}{2-q}, \quad U_{q}^{(1)}(t) \beta_{2-q}^{(1)}(t)=\frac{1}{2(2-q)}
$$

with

$$
z_{2-q}^{(1)}=\alpha \sqrt{\frac{2 \pi}{q-1}}\left(\frac{5-3 q}{2}\right)^{\frac{3-q}{2(1-q)}} \frac{\Gamma\left(\frac{1}{q-1}-\frac{1}{2}\right)}{\Gamma\left(\frac{1}{q-1}\right)},
$$

are equivalent to the solutions (2.13) $-(2.15)$, whilst the time evolution of $U_{q}^{(1)}(t)$ is still given by Eq. (2.15).

In a similar way, with the ansatz

$$
p^{(3)}(v, t)=\frac{1}{\bar{Z}_{q}^{(3)}(t)} \exp _{q}\left(-\beta_{q}^{(3)}(t)\left(\frac{1}{2} v^{2}-U_{q}^{(3)}(t)\right)\right)
$$

from Eq. (2.5) we obtain the system of the differential equations

$$
\frac{d}{d t} \ln X_{q}^{(3)}(t)+2 f_{q}^{(3)}(t)=0
$$

where $X_{q}^{(3)}(t)$ means $\left(\bar{Z}_{q}^{(3)}(t)\right)^{2}, 1 / \beta_{q}^{(3)}(t)$ and $U_{q}^{(3)}(t)$, respectively, and

$$
f_{q}^{(3)}(t)=1-D^{(1)}(2-q) \beta^{(3)}(t)\left(\bar{Z}_{q}^{(3)}(t)\right)^{2(q-1)}
$$

with $\beta^{(3)}(t)=\beta_{q}^{(3)}(t)\left(\bar{Z}_{q}^{(3)}(t)\right)^{1-q}$. We note that at equilibrium $\left(t=t_{\mathrm{e}}\right), \beta^{(3)}\left(t_{\mathrm{e}}\right)$ can be identified with the Lagrange multiplier of the escort mean energy $U_{q}^{(3)}\left(t_{\mathrm{e}}\right)$.

By using Eqs. (1.11) and (1.12), it is easy to verify that the system (2.22) can be transformed in the system (2.17) and therefore, it is also equivalent to the system (2.8), (2.9) and (2.12).

The solutions of Eqs. (2.22) are given by

$$
\left(\bar{Z}_{q}^{(3)}(t)\right)^{2} \beta_{q}^{(3)}(t)=\left(z_{q}^{(3)}\right)^{2}, \quad U_{q}^{(3)}(t) \beta_{q}^{(3)}(t)=\frac{1}{2}
$$


where

$$
z_{q}^{(3)}=\sqrt{\frac{2 \pi}{q-1}}\left(\frac{3-q}{2}\right)^{\frac{3-q}{2(1-q)}} \frac{\Gamma\left(\frac{1}{q-1}-\frac{1}{2}\right)}{\Gamma\left(\frac{1}{q-1}\right)},
$$

whilst the solution for the mean energy $U_{q}^{(3)}(t)$ has the same expression given in

Eq. (2.15) , with $U_{q}^{(1)} \rightarrow U_{q}^{(3)}$ and $D^{(1)} \rightarrow D^{(3)}$, being now $\mu_{0}=\alpha_{2}\left(\frac{3-q}{5-3 q}\right)^{\frac{q-3}{2}} U_{0}^{\frac{q-3}{2}}$ and $U_{0}=U_{q}^{(3)}(0)$.

\section{3 "Correspondence" between the modified 1st-formalism and the 3rd-formalism}

In this section, we study a different kind of relationship, which we call "correspondence", between the modified 1st-formalism and 3rd-formalism To start with, let us observe that by posing $q=2-1 / q^{\prime}$ through the definitions (2.20) and (2.25) we verify that

$$
z_{2-q}^{(1)}=z_{q^{\prime}}^{(3)}
$$

On the other hand, by assuming the same initial values for the both mean energy

$$
U_{q}^{(1)}(0)=U_{q^{\prime}}^{(3)}(0)
$$

and by comparing Eqs. (2.19) and (2.24) we get

$$
\beta_{2-q}^{(1)}(0)=q^{\prime} \beta_{q^{\prime}}^{(3)}(0), \quad \bar{Z}_{2-q}^{(1)}(0)=\bar{Z}_{q^{\prime}}^{(3)}(0)
$$

As a consequence, the following relation

$$
p_{q}^{(1)}(v, 0)=\left(\bar{Z}_{q^{\prime}}^{(3)}(0)\right)^{q^{\prime}-1}\left(p_{q^{\prime}}^{(3)}(v, 0)\right)^{q^{\prime}} \equiv p_{q^{\prime}}^{(\mathrm{E})}(v, 0)
$$

and its inverse

$$
p_{q^{\prime}}^{(3)}(v, 0)=\left(\bar{Z}_{2-q}^{(1)}(0)\right)^{1-q}\left(p_{q}^{(1)}(v, 0)\right)^{2-q}
$$


hold, where we denoted 2 with $p_{q^{\prime}}^{(\mathrm{E})}(v, 0)$ the escort distribution obtained from the optimization problem with the entropy $S_{q^{\prime}}^{\mathrm{E}}[p]$ and the corresponding linear mean energy $U_{q^{\prime}}^{(\mathrm{E})}$.

The consistency of Eqs. (3.4) and (3.5) can be easily verified as follows. First, by integrating Eq. (3.4) over $v$ and utilizing the normalization of $p_{q}^{(1)}(v, 0)$ and $p_{q^{\prime}}^{(3)}(v, 0)$, it reduces to the definition of the partition functions $\bar{Z}_{q^{\prime}}^{(3)}(0)$ and $\bar{Z}_{2-q}^{(1)}(0)$. Second, by multiplying Eq. (3.4) by $\epsilon(v)$ and integrating over $v$, we can verify that

$$
U_{q}^{(1)}(0)=\int \epsilon(v) p_{q}^{(1)}(v, 0) d v=\frac{\int \epsilon(v)\left(p_{q^{\prime}}^{(3)}(v, 0)\right)^{q^{\prime}} d v}{\int\left(p_{q^{\prime}}^{(3)}(v, 0)\right)^{q^{\prime}} d v}=U_{q^{\prime}}^{(3)}(0)
$$

which is the initial condition (3.2).

All of these results are already known for the equilibrium system. Notwithstanding, let us now pose the following question: Does the "correspondence" established through Eqs. (3.4) and (3.5) still hold, in some sense, when the system evolves toward the equilibrium according to a suitable NFPEs?

For reasons which will be clarified through this section, it is more appropriate to start by studying the "correspondence" among $p_{q}^{(1)}(v, t)$ and $p_{q^{\prime}}^{(\mathrm{E})}(v, t)$ and only successively recover the "correspondence" with $p_{q^{\prime}}^{(3)}(v, t)$ by means of Eq. (3.4). In fact, by employing the escort-formalism we get the advantage to deal with a linear expression for the mean energy $U_{q^{\prime}}^{(\mathrm{E})}(t) \equiv U_{q^{\prime}}^{(3)}(t)=$ $\int \epsilon(v) p_{q^{\prime}}^{(\mathrm{E})}(v, t) d v$. In this way, by posing

$$
\mathcal{L}=\mathcal{L}_{q^{\prime}}^{(\mathrm{E})}[p] \equiv U_{q^{\prime}}^{(\mathrm{E})}(t)-D^{(\mathrm{E})} S_{q^{\prime}}^{(\mathrm{E})}[p]
$$

where $S_{q}^{(\mathrm{E})}[p]$ is the escort entropy (1.13), we derive the following kinetic equation:

$$
\frac{\partial}{\partial t} p_{q^{\prime}}^{(\mathrm{E})}(v, t)=\frac{\partial}{\partial v}\left[v p_{q^{\prime}}^{(\mathrm{E})}(v, t)+D^{(\mathrm{E})}\left(\bar{Z}_{q^{\prime}}^{(\mathrm{E})}(t)\right)^{\frac{1}{q^{\prime}}-q^{\prime}} \frac{\partial}{\partial v}\left(p_{q^{\prime}}^{(\mathrm{E})}(v, t)\right)^{\frac{1}{q^{\prime}}}\right]
$$

where $\bar{Z}_{q^{\prime}}^{(E)}(t)=\bar{Z}_{q^{\prime}}^{(3)}(t)=\left(\int p_{q^{\prime}}^{(\mathrm{E})}(v, t)^{1 / q^{\prime}} d v\right)^{q^{\prime} /\left(q^{\prime}-1\right)}$ and $D^{(E)}$ is the constant diffusion coefficient for the given problem. This equation, for $q=1 / q^{\prime}$, becomes equivalent to the NFPE obtained previously in [26].

Let us observe that Eqs. (2.1) and (2.2) provide a general mechanism to construct NFPEs starting from a give Lyapunov functional $\mathcal{L}[p]$. This method

$\overline{2}$ From now on, for the sake of clarity, we indicate explicitly the dependence on the parameter $q$ ( $q^{\prime}$ respectively) in the distribution. 
works also when the mean energy term in the Lyapunov functional has a nonlinear dependence on the probability distribution functions like, for instance, in the case of $U_{q}^{(3)}$. Notwithstanding, in these cases the resulting nonlinear current $J(v, t)$ will contain, among to a nonlinear diffusion term, also a nonlinear drift term. This fact makes problematic the comparison between the different formalisms which is the main purpose of the present work. A possible way to avoid such complication is actually furnished by means of the escort formalism centered on the linear definition for the mean energy $U_{q}^{(\mathrm{E})}$.

Come back to the original question, self-similar solutions of Eq. (3.8) can be obtained by posing

$$
p_{q^{\prime}}^{(\mathrm{E})}(v, t)=\frac{1}{\bar{Z}_{q^{\prime}}^{(E)}(t)}\left[1-\left(1-q^{\prime}\right) \beta_{q^{\prime}}^{(\mathrm{E})}(t)\left(\frac{1}{2} v^{2}-U_{q^{\prime}}^{(\mathrm{E})}(t)\right)\right]^{\frac{q^{\prime}}{1-q^{\prime}}},
$$

where $\beta_{q^{\prime}}^{(\mathrm{E})}(t)=\beta^{(\mathrm{E})}(t)\left(\bar{Z}_{q^{\prime}}^{(\mathrm{E})}(t)\right)^{\frac{1}{q^{\prime}}-1}$ and at equilibrium $\beta^{(\mathrm{E})}\left(t_{\mathrm{e}}\right)$ is the Lagrange multiplier related to the escort mean energy $U_{q^{\prime}}^{(\mathrm{E})}$.

We get the following system of equations

$$
\frac{\partial}{\partial t} \ln X_{q^{\prime}}^{(\mathrm{E})}(t)+2 f_{q^{\prime}}^{(\mathrm{E})}(t)=0
$$

where $X_{q}^{(\mathrm{E})}(t)$ means $\left(\bar{Z}_{q}^{(\mathrm{E})}(t)\right)^{2}, 1 / \beta_{q}^{(\mathrm{E})}(t)$ and $U_{q}^{(\mathrm{E})}(t)$, respectively, and

$$
f_{q}^{(\mathrm{E})}(t)=1-D^{(\mathrm{E})} \beta^{(\mathrm{E})}(t)\left(\bar{Z}_{q}^{(\mathrm{E})}(t)\right)^{\frac{1}{q}-q} .
$$

The solution of the system (3.10) can be easily written in the form

$$
\left(\bar{Z}_{q^{\prime}}^{(\mathrm{E})}(t)\right)^{2} \beta_{q^{\prime}}^{(\mathrm{E})}(t)=\left(z_{q^{\prime}}^{(3)}\right)^{2}, \quad U_{q^{\prime}}^{(\mathrm{E})}(t) \beta_{q^{\prime}}^{(\mathrm{E})}(t)=\frac{1}{2},
$$

and

$$
U_{q^{\prime}}^{(\mathrm{E})}(t)=U_{0}\left[\left(1-D^{(\mathrm{E})} \mu_{0}^{(\mathrm{E})}\right) e^{-\left(1+q^{\prime}\right) t}+D^{(\mathrm{E})} \mu_{0}^{(\mathrm{E})}\right]^{\frac{2}{1+q^{\prime}}}
$$

with $\mu_{0}^{(\mathrm{E})}=2^{-\frac{1+q^{\prime}}{2}}\left(z_{q^{\prime}}^{(3)}\right)^{1-q^{\prime}} U_{0}^{\frac{1-q^{\prime}}{2}}$

Let us now introduce the two different time-scales ( $t$ and $\left.t^{\prime}\right)$ according to the relation

$$
\frac{d t^{\prime}}{d t}=\frac{f_{q}^{(1)}(t)}{f_{q^{\prime}}^{(\mathrm{E})}\left(t^{\prime}\right)}
$$


Accounting for the expressions (2.18) and (3.11), Eq. (3.14) can be integrated in

$$
t^{\prime}=t_{0}-\frac{1}{1+q^{\prime}} \ln \left\{\left[\left(1-D^{(1)} \mu_{0}^{(1)}\right) e^{-\left(\frac{1}{q^{\prime}}+1\right) t}+D^{(1)} \mu_{0}^{(1)}\right]^{q^{\prime}}-D^{(\mathrm{E})} \mu_{0}^{(\mathrm{E})}\right\},
$$

where

$$
t_{0}=\frac{1}{1+q^{\prime}} \ln \left(1-D^{(\mathrm{E})} \mu_{0}^{(\mathrm{E})}\right) .
$$

By converting the time-scale $t$ into $t^{\prime}$, we can easily verify that the set of equations for the modified 1st formalism are transformed to those for the escort formalism

$$
\frac{d}{d t} \ln X_{q}^{(1)}(t)+f_{q}^{(1)}(t)=0, \quad \Rightarrow \quad \frac{d}{d t^{\prime}} \ln X_{q^{\prime}}^{(\mathrm{E})}\left(t^{\prime}\right)+f_{q^{\prime}}^{(\mathrm{E})}\left(t^{\prime}\right)=0,
$$

which implies the following "correspondence"

$$
X_{q}^{(1)}(t)=X_{q^{\prime}}^{(\mathrm{E})}\left(t^{\prime}\right)
$$

Since the quantities $X_{q}^{(1)}(t)$ and $X_{q^{\prime}}^{(\mathrm{E})}\left(t^{\prime}\right)$ determine completely the shape of the distribution $p_{q}^{(1)}(v, t)$ and that of $p_{q^{\prime}}^{(\mathrm{E})}\left(v, t^{\prime}\right)$, respectively, we conclude that the "correspondence" stated in Eq. (3.4) at the initial time actually holds for any later time, i.e.

$$
p_{q}^{(1)}(v, t)=p_{q^{\prime}}^{(\mathrm{E})}\left(v, t^{\prime}\right) .
$$

Finally, taking into account the expressions (2.16) and (3.9), from the relation (3.19) we obtain

$$
\begin{aligned}
& \frac{\partial}{\partial t} p_{q}^{(1)}(v, t)=\frac{\partial}{\partial v}\left[v p_{q}^{(1)}(v, t)+D^{(1)} \frac{\partial}{\partial v}\left(p_{q}^{(1)}(v, t)\right)^{2-q}\right] \Rightarrow \\
& \frac{\partial}{\partial t^{\prime}} p_{q^{\prime}}^{(\mathrm{E})}\left(v, t^{\prime}\right)=\frac{\partial}{\partial v}\left[v p_{q^{\prime}}^{(\mathrm{E})}\left(v, t^{\prime}\right)+D^{(\mathrm{E})}\left(\bar{Z}_{q^{\prime}}^{(\mathrm{E})}\left(t^{\prime}\right)\right)^{\frac{1}{q^{\prime}}-q^{\prime}} \frac{\partial}{\partial v}\left(p_{q^{\prime}}^{(\mathrm{E})}\left(v, t^{\prime}\right)\right)^{\frac{1}{q^{\prime}}}\right]
\end{aligned}
$$

i.e., concerning self-similar solutions, both the NFPEs (2.5) and (3.8) describe the same kinetic process when considered in the own time-scale $t$ and $t^{\prime}$, respectively. 
In figure 1 , we plot the relevant quantities $U_{q}^{(1)}(t)$ [resp. $\left.U_{q^{\prime}}^{(\mathrm{E})}\left(t^{\prime}\right)\right], \bar{Z}_{2-q}^{(1)}(t)$ [resp. $\left.\bar{Z}_{q^{\prime}}^{(\mathrm{E})}\left(t^{\prime}\right)\right], \beta_{2-q}^{(1)}(t)\left[\right.$ resp. $\left.\beta_{q^{\prime}}^{(\mathrm{E})}\left(t^{\prime}\right)\right]$ and $p_{q}^{(1)}(0, t)\left[\right.$ resp. $\left.p_{q^{\prime}}^{(\mathrm{E})}\left(0, t^{\prime}\right)\right]$, for the case $q=1.4 ; D^{(1)}=0.2$ and $D^{(\mathrm{E})}=1$. In all panels the full line depicts the evolution of these quantities in the modified 1st-formalism v.s. time $t$, whilst the dashed line depicts the evolution of the same quantities in the escortformalism v.s. the time $t$. Finally, the dotted line depicts the evolution of all these quantities in the escort-formalism v.s. the transformed time $t^{\prime}$. We can see that all the functions in the escort-formalism, referred to the time $t^{\prime}$, coincide with the corresponding ones in the modified 1st-formalism, referred to the time $t$ for all time 3 .
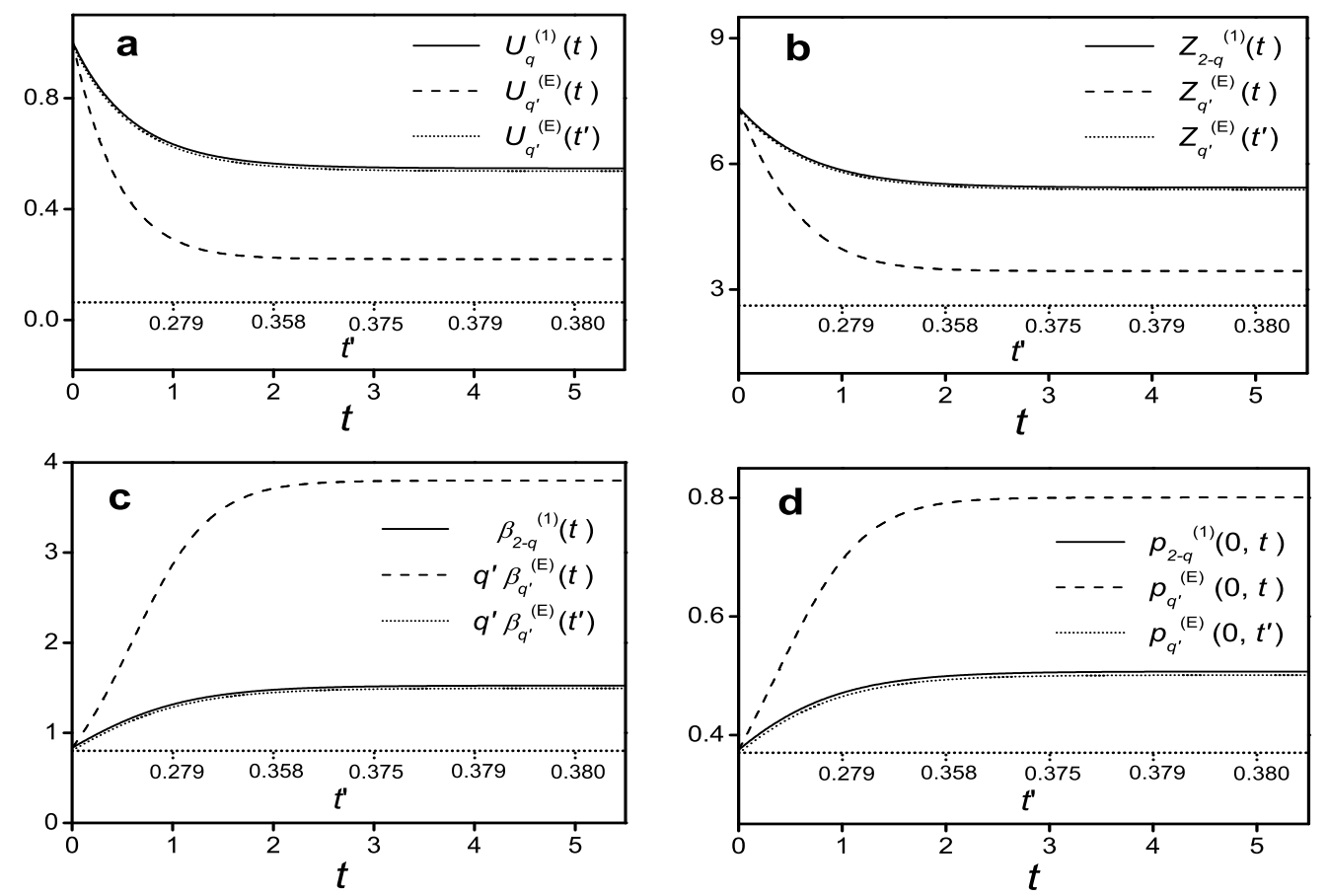

Fig. 1. Plots, in arbitrary unity, of: a) $U_{q}^{(1)}(t)\left[\right.$ resp. $\left.U_{q^{\prime}}^{(\mathrm{E})}\left(t^{\prime}\right)\right]$, b) $\bar{Z}_{2-q}^{(1)}(t)$ [resp. $\left.\bar{Z}_{q^{\prime}}^{(\mathrm{E})}\left(t^{\prime}\right)\right]$, c) $\beta_{2-q}^{(1)}(t)\left[\operatorname{resp} . \beta_{q^{\prime}}^{(\mathrm{E})}\left(t^{\prime}\right)\right]$, d) $p_{q}^{(1)}(0, t)\left[\right.$ resp. $\left.p_{q^{\prime}}^{(\mathrm{E})}\left(0, t^{\prime}\right)\right]$. Full line: modified 1 st-formalism v.s. time $t$; dashed line: escort-formalism v.s. time $t$; dotted line: escort-formalism v.s. time $t^{\prime}$.

$\overline{3}$ Note that in the figure we have slightly shifted, by hand, the dotted curves for the sake of presentation. 
As known, some concepts like irreversibility of the process described by the Fokker-Planck equation or the $H$-theorem ensuring uniqueness and stability of equilibrium distribution, can be well described by means of the Lyapunov function. Existence of a Lyapunov function for Eqs. (2.5) and (3.8) in connection with the $H$-theorem has been investigated in [26] and more in general, for an arbitrary NFPE, in [23]. In the following, we make some considerations concerning the "correspondence" among the Lyapunov functionals $\mathcal{L}_{q}^{(1)}[p]$ and $\mathcal{L}_{q^{\prime}}^{(\mathrm{E})}[p]$ arising in the two formalisms.

Firstly, we observe that the both functionals $\mathcal{L}_{q}^{\mathrm{i}}[p]$ given in Eqs. (2.3) and (3.7), where $\mathrm{i}=(1)$ or $(\mathrm{E})$, are actually Lyapunov functionals for the kinetic equations (2.5) and (3.8), respectively, since they fulfill the relation

$$
\frac{d \mathcal{L}_{q}^{\mathrm{i}}}{d t}=-2 U^{\mathrm{i}}(t)\left(f_{q}^{\mathrm{i}}(t)\right)^{2} \leq 0
$$

where equality holds at equilibrium $\left(f_{q}^{\mathrm{i}}\left(t_{\mathrm{e}}\right)=0\right)$. According to Eq. (3.18) we have

$$
\left(f_{q^{\prime}}^{(\mathrm{E})}\left(t^{\prime}\right)\right)^{-2} \frac{d \mathcal{L}_{q^{\prime}}^{(\mathrm{E})}}{d t^{\prime}}=\left(f_{q}^{(1)}(t)\right)^{-2} \frac{d \mathcal{L}_{q}^{(1)}}{d t}
$$

which relates the variation in time of the Lyapunov functions in both formalisms.

By recalling Eqs. (2.17) and (3.10), with $X_{q}^{\mathrm{i}}(t)=U_{q}^{\mathrm{i}}(t)$, we can rewrite Eq. (3.21) in the form

$$
\frac{d \mathcal{L}_{q}^{\mathrm{i}}}{d t}=\frac{d U_{q}^{\mathrm{i}}}{d t} f_{q}^{\mathrm{i}}(t)
$$

so that

$$
\frac{d \mathcal{L}_{q}^{\mathrm{i}}}{d U_{q}^{\mathrm{i}}}=f_{q}^{\mathrm{i}}(t)
$$

i.e., the functions $f_{q}^{\mathrm{i}}(t)$, responsible for the different time-scale in the two formalisms, originate from the variation of the Lyapunov function with respect to the mean energy.

Finally, let us remind that the distributions $p_{q^{\prime}}^{(3)}\left(v, t^{\prime}\right)$ and $p_{q^{\prime}}^{(\mathrm{E})}\left(v, t^{\prime}\right)$ are closely related to each other according to Eq. (3.4). By inserting this transformation into Eq. (3.8) we obtain the following kinetic equation for the distribution $p_{q^{\prime}}^{(3)}(v, t)$ 


$$
\frac{\partial}{\partial t^{\prime}} p_{q^{\prime}}^{(3)}\left(v, t^{\prime}\right)=\frac{\partial}{\partial v}\left[v p_{q^{\prime}}^{(3)}\left(v, t^{\prime}\right)+\frac{D^{(1)}}{2-q^{\prime}}\left(\bar{Z}_{q^{\prime}}^{(3)}\right)^{2\left(1-q^{\prime}\right)} \frac{\partial}{\partial v}\left(p_{q^{\prime}}^{(3)}\left(v, t^{\prime}\right)\right)^{2-q^{\prime}}\right]
$$

where we assumed $D^{(1)} \equiv D^{(\mathrm{E})}$. By means of the relation

$$
D^{(3)}\left(t^{\prime}\right)=\frac{D^{(1)}}{2-q^{\prime}}\left(\bar{Z}_{q^{\prime}}^{(3)}\left(t^{\prime}\right)\right)^{2\left(1-q^{\prime}\right)}
$$

equation (3.25) assumes the same expression of Eq. (2.5), but with a time dependent diffusion coefficient.

It is worthy to observe that, contrarily to the NFPEs (2.5) and (3.8), Eq. (3.25) does not follow from Eqs. (2.1) and (2.2) and it has been derived just on the basis of the "correspondence" between self-similar solutions in the different formalism. From a formal point of view, we can state that kinetic evolution of self-similar solutions, both in the $(2-q)$-formalism and in the 3rd-formalism, are described by the same NFPE with a constant diffusion coefficient $D^{(1)}$ in the former case and a time dependent diffusion coefficient $D^{(3)}\left(t^{\prime}\right)$ in the later case (or vice versa). Remarkably, since $1 / D^{(1)}=\beta^{(1)}$ and at equilibrium $1 / D^{(3)}\left(t_{\mathrm{e}}\right)=\beta^{(3)}$, Eq. (3.26) reduces to Eq. (1.11) with $q$ replaced by $q^{\prime}$.

By following the same steps described at the begin of this section, by replacing the relation

$$
\frac{\partial}{\partial v}\left(p_{q}^{(3)}(v, t)\right)^{2-q}=-v(2-q) \beta^{(3)}(t) p_{q}^{(3)}(v, t)
$$

in Eq. (3.25), it can be rewritten in the form

$$
\frac{\partial}{\partial t^{\prime \prime}} p_{q^{\prime}}^{(3)}\left(v, t^{\prime \prime}\right)=\left(1-D^{(3)} \beta^{(3)}\left(t^{\prime \prime}\right)\left(\bar{Z}_{q^{\prime}}^{(3)}\left(t^{\prime \prime}\right)\right)^{2\left(1-q^{\prime}\right)}\right) \frac{\partial}{\partial v}\left(v p_{q^{\prime}}^{(3)}\left(v, t^{\prime \prime}\right)\right) .
$$

In this way, by introducing the (time-evolution) function

$$
f_{q^{\prime}}^{(3)}\left(t^{\prime \prime}\right)=1-D^{(3)} \beta^{(3)}\left(t^{\prime \prime}\right)\left(\bar{Z}_{q^{\prime}}^{(3)}\left(t^{\prime \prime}\right)\right)^{2\left(1-q^{\prime}\right)}
$$

we can establish a "correspondence" between self-similar solutions in the modified 1st-formalism and those of the 3rd-formalism, in the sense that, according to the transformation

$$
p_{q^{\prime}}^{(3)}\left(v, t^{\prime \prime}\right)=\left(\bar{Z}_{2-q}^{(1)}(t)\right)^{1-q}\left(p_{q}^{(1)}(v, t)\right)^{2-q}
$$


and

$$
\frac{d t^{\prime \prime}}{d t}=\frac{f_{q}^{(1)}(t)}{f_{q^{\prime}}^{(3)}\left(t^{\prime \prime}\right)}
$$

the NFPE (3.25) turns into Eq. (2.5).

\section{Conclusions}

In the present letter, we have compared self-similar solutions of some NFPEs obtained from different generalized entropies. For the sake of simplicity we assumed a quadratic form of the energy density but extension to a more general expression $\epsilon(v)=k_{0}+k_{1} v+k_{2} v^{2}$, with $k_{i}>0$, is straightforward.

Our results can be summarized in the following two main points.

1) According to Eqs. (1.11) and (1.12) we have shown that solutions $p(v, t)$ of the NFPE (2.5), derived in the $(2-q)$-formalism, can be written in the form $p_{q}^{(1)}(v, t)$ obtained in the modified 1st-formalism or in the form $p_{q}^{(3)}(v, t)$ obtained in the 3rd-formalism.

2) We have shown that the solution $p_{q}^{(1)}(v, t)$ of the NFPE (2.5) "corresponds" to the solution $p_{q^{\prime}}^{(\mathrm{E})}(v, t)$ of the NFPE (3.8) derived in the escort-formalism, where $q=2-1 / q^{\prime}$. Remark that this relation embodies both dualities $q \rightarrow 2-q$ and $q \rightarrow 1 / q$. These two transformations appear recurrently, alone or combined, in the framework of the generalized statistical mechanics based on the Tsallis entropy [33,34,35,36,37,38].

The "correspondence" $p_{q}^{(1)}(v, t)=p_{q^{\prime}}^{(\mathrm{E})}\left(v, t^{\prime}\right)$ is established by means of different time-scale, stated through Eq. (3.14). As a consequence, it has been shown that the (self-similar) solutions, obtained in the modified 1st-formalism and the ones obtained in the 3rd-formalism, obey to similar kinetic equations where the diffusion coefficients are related according to Eq. (3.26).

This paper makes some progress about the still discussed question of the equivalence between the various formalisms, introduced in the generalized statistical mechanics. We have shown that the formulation of the theory centered around the escort mean values (1.4), whose physical meaning is still unclear at all, are in any way valid, being related to the same results obtained by employing the orthodox mean value definition (1.2). This encourages the use of the escort probabilities which, in some cases, expedites the derivation of several results concerning this theory.

On a general basis, we have verified that concerning the class of self-similar solutions, there are some different nonlinear diffusive equations which describe the same kinetic process when it is observed in the appropriate time-scale. The time-scale is derived by means of the Lyapunov function which generates the corresponding kinetics evolution equation. In this way, by modifying the 
expression of the Lyapunov function, we can produce a family of nonlinear Fokker-Planck equations belonging to the same equivalent class, in the sense discussed in this work. It should be interesting to verify the existence of these equivalences also for more general kind of solutions.

\section{Appendix A}

We summarize, for the sake of comparison, the expressions of some physically relevant quantities like the entropy, the mean energy constraint and the distribution for the different formalisms discussed in this paper.

All these quantities are related by means of the following variational principle

$$
\frac{\delta}{\delta p}\left(S[p]-\gamma \Phi_{0}[p]-\beta \Phi_{1}[p]\right)=0
$$

where $\gamma$ and $\beta$ are the Lagrange multipliers related to the constraints (given through $\Phi_{0}[p]$ and $\left.\Phi_{1}[p]\right)$ of the normalization and that of the mean energy, respectively.

a) $(2-q)$-formalism.

$$
\begin{aligned}
& S_{2-q}[p]=\frac{1}{q-1} \int\left[p(v)^{2-q}-p(v)\right] d v \\
& \Phi_{1}[p]=\int \epsilon(v) p(v) d v-U_{q} \\
& p(v)=\alpha \exp _{q}(-\gamma-\beta \epsilon(v)) .
\end{aligned}
$$

b) Modified 1st-formalism.

$$
\begin{aligned}
& S_{q}[p]=\frac{1}{1-q} \int\left[\left(p^{(1)}(v)\right)^{q}-p^{(1)}(v)\right] d v \\
& \Phi_{1}[p]=\int \epsilon(v) p^{(1)}(v) d v-U_{q}^{(1)} \\
& p^{(1)}(v)=\frac{1}{\bar{Z}_{q}^{(1)}} \exp _{2-q}\left(-\beta_{q}^{(1)}\left(\epsilon(v)-U_{q}^{(1)}\right)\right), \\
& \beta_{q}^{(1)}=\frac{\beta}{q}\left(\bar{Z}_{q}^{(1)}\right)^{q-1} \\
& \bar{Z}_{q}^{(1)}=q^{1 /(q-1)} \exp _{q}\left(\gamma+\beta U_{q}^{(1)}\right) .
\end{aligned}
$$


c) 2nd-formalism.

$$
\begin{aligned}
& S_{q}[p]=\frac{1}{1-q} \int\left[\left(p^{(2)}(v)\right)^{q}-p^{(2)}(v)\right] d v \\
& \Phi_{1}[p]=\int \epsilon(v)\left(p^{(2)}(v)\right)^{q} d v-U_{q}^{(2)} \\
& p^{(2)}(v)=\frac{1}{Z_{q}^{(2)}} \exp _{q}(-\beta \epsilon(v)) \\
& Z_{q}^{(2)}=q^{1 /(q-1)} \exp _{q}(\gamma)
\end{aligned}
$$

d) 3rd-formalism.

$$
\begin{aligned}
& S_{q}[p]=\frac{1}{1-q} \int\left[\left(p^{(3)}(v)\right)^{q}-p^{(3)}(v)\right] d v \\
& \Phi_{1}[p]=\frac{\int \epsilon(v)\left(p^{(3)}(v)\right)^{q} d v}{\int\left(p^{(3)}(v)\right)^{q} d v}-U_{q}^{(3)} \\
& p^{(3)}(v)=\frac{1}{\bar{Z}_{q}^{(3)}} \exp _{q}\left(-\beta_{q}^{(3)}\left(\epsilon(v)-U_{q}^{(3)}\right)\right) \\
& \beta_{q}^{(3)}=\beta\left(\bar{Z}_{q}^{(3)}\right)^{q-1} \\
& \bar{Z}_{q}^{(3)}=q^{1 /(q-1)} \exp _{q}(\gamma) .
\end{aligned}
$$

e) OLM-formalism.

$$
\begin{aligned}
& S_{q}[p]=\frac{1}{1-q} \int\left[\left(p^{(\mathrm{OLM})}(v)\right)^{q}-p^{(\mathrm{OLM})}(v)\right] d v \\
& \Phi_{1}[p]=\int \epsilon(v)\left(p^{(\mathrm{OLM})}(v)\right)^{q} d v-U_{q}^{(\mathrm{OLM})} \int\left(p^{(\mathrm{OLM})}(v)\right)^{q} d v \\
& p^{(\mathrm{OLM})}(v)=\frac{1}{\bar{Z}_{q}^{(\mathrm{OLM})}} \exp _{q}\left(-\beta\left(\epsilon(v)-U_{q}^{(\mathrm{OLM})}\right)\right), \\
& \bar{Z}_{q}^{(\mathrm{OLM})}=q^{1 /(q-1)} \exp _{q}(\gamma) .
\end{aligned}
$$

f) Escort-formalism.

$$
\begin{aligned}
& S_{q}^{(\mathrm{E})}[p]=\frac{1}{1-q}\left\{\frac{\int p^{(\mathrm{E})}(v) d v}{\left[\int\left(p^{(\mathrm{E})}(v)\right)^{1 / q} d v\right]^{q}}-1\right\}, \\
& \Phi_{1}[p]=\int \epsilon(v) p^{(\mathrm{E})}(v) d v-U_{q}^{(\mathrm{E})},
\end{aligned}
$$




$$
\begin{aligned}
& p^{(\mathrm{E})}(v)=\frac{1}{\bar{Z}_{q}^{(E)}}\left[\exp _{q}\left(-\beta_{q}^{(\mathrm{E})}\left(\epsilon(v)-U_{q}^{(\mathrm{E})}\right)\right)\right]^{q}, \\
& \beta_{q}^{(\mathrm{E})}=\beta\left(\bar{Z}_{q}^{(\mathrm{E})}\right)^{q-1}, \\
& \gamma=-\beta U_{q}^{(\mathrm{E})} .
\end{aligned}
$$

\section{References}

[1] C. Tsallis, J. Stat. Phys. 52, 479 (1988).

[2] http://tsallis.cat.cbpf.br/biblio.htm

[3] R.P. Di Sisto, S. Martínez, R.B. Orellana, A.R. Plastino, A. Plastino, Physica A 265 (1999) 590-613.

[4] A.G. Bashkirov, Physica A 340, 153 (2000).

[5] E.M.F. Curado, C. Tsallis, J. Phys. A 24, L69 (1991); Corrigenda: 24, 3187 (1991) and 25, 1019 (1992).

[6] C. Tsallis, R.S. Mendes, A.R. Plastino, Physica A 261, 534 (1998).

[7] S. Martínez, F. Nicolás, F. Pennini, A. Plastino, Physica A 286, 489 (2000).

[8] E.K. Lenzi. R.S. Mendes, L.R. da Silva, Physica A 280, 337 (2000).

[9] G.L. Ferri, S. Martinez, A. Plastino, Physica A 347, 205 (2005).

[10] G. Kaniadakis, M. Lissia, A.M. Scarfone, Physica A 340, 41 (2004); Phys. Rev. E 71, 046128 (2005).

[11] A.M. Scarfone, T. Wada, Phys. Rev. E 71, 051103 (2005).

[12] T. Wada, A.M. Scarfone, Eur. Phys. J. B 47, 557 (2005).

[13] T. Wada, A.M. Scarfone, Phys. Lett. A 335, 351 (2005).

[14] G.L. Ferri, S. Martinez, A. Plastino, J. Stat. Mech.: Theor. \& Exp. P04009, Apr. (2005).

[15] C. Tsallis, E. Brigatti, Continuum Mech. Thermodyn. 16, 223 (2004).

[16] J. Stephenson, Physica A 222, 234 (1995).

[17] H. Pascal, Physica D 39, 262 (1989); Physica A 197, 593 (1993).

[18] J.P. Pascal, H. Pascal, Physica A 208, 351 (1994).

[19] G. Kaniadakis, G. Lapenta, Phys. Rev. E 62, 3246 (2000).

[20] A. Compte, D. Jou, Y. Katayama J. Phys. A: Math. Gen. 30, 1023 (1997). 
[21] A. Compte, D. Jou, J. Phys. A: Math. Gen. 29, 4321 (1996).

[22] T.D. Frank, A. Daffertshofer, Physica A 272, 497 (1999).

[23] T.D. Frank, Phys. Lett. A 290, 93 (2001); Physica A 310, 397 (2002).

[24] G. Kaniadakis, Physica A 296, 405 (2001).

[25] A.R. Plastino, A. Plastino, Physica A 222, 347 (1995).

[26] M. Shiino, J. Phys. Soc. Jpn. 67, 3658 (1998); J. Math. Phys. 42, 2540 (2001); Phys. Rev. E 67, 056118 (2003).

[27] T.D. Frank, Nonlinear Fokker-Planck Equations: Fundamentals and Applications, (Springer Series in Synergetics, Berlin, 2005).

[28] L. Borland, Phys. Rev. E 57, 6634 (1998).

[29] G. Drazer, H.S. Wio, C. Tsallis, Phys. Rev. E 61, 1417 (2000).

[30] E. K. Lenzi, C. Anteneodo, L. Borland, Phys. Rev. E 63, 051109 (2001).

[31] C. Tsallis, Physica A 365, 7 (2006).

[32] C. Tsallis, D.J. Bukman, Phys. Rev. E 54, R2197 (1996).

[33] C. Tsallis, Prog. Theor. Phys. Suppl. 162, 1 (2006).

[34] A. Robledo, Physica D 193, 153 (2004).

[35] E. Mayoral, A. Robledo, Phys. Rev. E 72, 026209 (2005).

[36] L.F. Burlaga, A.F. Viñas, Physica A 356, 375 (2005).

[37] S. Umarov, C. Tsallis, M. Gell-Mann, S. Steinberg, q-generalization of symmetric alpha-stable distribution. Part II, arXiv:cond-mat/0606040.

[38] L.G. Moyano, C. Tsallis, M. Gell-Mann, Europhys. Lett. 73, 813 (2006). 